\title{
Antagonistic activity and Biofilm studies on Streptomyces species towards Nigrospora sp
}

\author{
Sudhakar Malla*, Bathula Christopher, Yacharam shivakumar, Kakarapalli Nagalakshmi \\ Indian Academy Degree College, Centre for Research \& Post Graduate Studies, Bangalore, India \\ Email address: \\ sudhasanmoon2000@gmail.com (S. Malla)
}

To cite this article:

Sudhakar Malla, Bathula Christopher, Yacharam shivakumar, Kakarapalli Nagalakshmi. Antagonistic Activity and Biofilm Studies on Streptomyces Species towards Nigrospora Sp. American Journal of Life Sciences. Special Issue: Recent Developments in Health Care through Plants and Microbes. Vol. 2, No. 6-1, 2014, pp. 28-34. doi: 10.11648/j.ajls.s.2014020601.16

\begin{abstract}
Plants, in contrast with vertebrates, have no immune system. So they are affected by pathogens easily leading to remarkable yield loss altogether. Fungal phytopathogens pose serious problems worldwide in the agriculture sector, and harmful and costly chemical fungicides are nowhere a match to them. A detailed study was performed to screen the cooperative role of Streptomyces coelicolor and Streptomyces halstedii towards the fungal pathogen Nigrospora, a predominant pathogen of banana plantation. Many works were published stating the antagonistic activity of the Streptomyces species. For the first time we tried to study against the Nigrospora, which is a predominant pathogen of banana plant. Moreover we tried to find out the cooperative role of both the species towards the fungal pathogen. Biofilm formation studies were done using the ethanol method, and Chitinase activity was quantified using DNS method. Free radical scavenging activity was quantified using the standard DPPH method. The antioxidant profiles were screened using the TLC plate in mobile phase (5:4:3 (v/v/v) n-butanol/Methanol/16\% aqueous ammonia). The antagonistic screening test done using the cup plate method proved the cooperative role of both the species. The activity of chitinase was observed for all the groups. Both the species showed chitinase activity, but when they are co cultured the activity was found to be enhanced. Even the co culture study also proved of the strong biofilm formation. Previous literature also showed of the exhibition of biofilm formation of the Streptomyces species. The increase or stability in the values proves of the possible cooperative role of both the species in the antagonistic activity against the Nigrospora species. A one way ANOVA was done to show the significance in the formation of biofilms. Peculiar results were obtained in the TLC. The Rf values obtained were compared with the standard antioxidant $\mathrm{Rf}$ values. The $\mathrm{Rf}$ values of 0.12 match with the catechin, and 0.4 match with the Quercetin, 06 match with Caffeic acid.
\end{abstract}

Keywords: Stretomyces Coelicolor, Streptmyces Halstedii, Antifungal Antagonistic Activity, Nigrospora, TLC

\section{Introduction}

Chemical fungicides are extensively adopted in current of plant diseases. Therefore, biological control tactics become an important approach to facilitate sustainable agriculture. Farming practices were modified now and then to protect the crops from diseases. However, recently their utilization has attracted increased scrutiny since chemical fungicides are highly toxic, and can cause environmental pollution, and may induce pathogen resistance [1].

Photosynthetic organisms form the basis of all food webs and higher land plants are the primary energy and carbon source for terrestrial ecosystems to feed the human population. However, all of the approximately 300,000 plant species regularly suffer pathogen and herbivore attacks $[2,3]$. Thus, the need for a systematic and easy comprehensive understanding of the negative impacts of pathogens on plants is evident.

After infection, when the pathogen has established itself in the host, it will heavily depend on host metabolism and, as a consequence, the metabolism of pathogen and host become tightly interlinked. This generally imposes severe nutrient losses of the plant to the pathogen [4].

The organism that suppresses the pest or pathogen is referred to as the biological control agents (BCA). According to the members of the U.S. National Research Council, 'Biological control is the use of natural or modified organisms, gene, or gene products, to reduce the effect of undesirable organisms and to favor desirable 
organisms such as crops, beneficial insects, and microorganisms.' Plants and pathogens interact with a wide variety of microorganisms. These interactions can significantly affect plant health in various ways $[5,6]$.

Control of the soil borne pathogens with chemicals is practically difficult. On the other hand, indiscriminate use of chemicals causes environmental pollution and health hazards [7, 8]. Nowadays, integrated Disease Management (IDM) is very much popular for controlling plant diseases. There are several tactics within IDM, among them biological control being one of the most important tactics. The use of antagonistic bacteria as a biological control means may provide a great alternative for plant pathologists [8]

In the past century, farmers often relied heavily on chemical fertilizers and pesticides to increase the productivity of plants. However, the environmental pollution caused by excessive use and misuse of agrochemicals, as well as concern by some opponents of pesticides, has led to considerable changes in people's attitude toward the use of pesticides in agriculture [9]. Consequently, some pest management researchers have focused their efforts on developing alternative inputs to synthetic chemicals for control pests and diseases. Some microorganisms nearby rhizosphere help plant health from pathogen attack, directly or indirectly [10].

In recent years, the search for microorganisms antagonistic toward fungi has intensified due to the fact that they are causative factors of many plant diseases. This is usually associated with the production of antifungal compounds and extracellular hydrolytic enzymes (chitinase and 1,3-ßglucanase). Chitinolytic enzymes are able to lyse the cell wall of many fungi. The microorganisms that produce these chitynolytic enzymes are able to destroy the cell wall of many fungi [11]. The microorganisms that produce these enzymes are capable of eradicating fungal diseases that are a problem for global agricultural production. Chitynolytic microorganisms may be an alternative to chemical agents and could be employed as natural plant protection methods against fungal diseases [12]. To this end, microorganisms able to synthesize compounds that naturally inhibit the growth of phytopathogenic fungi are being searched for. Compared to synthetic fungicides, they do not contaminate the environment, this being a crucial factor in increased interest in the use of biological methods to combat plant pathogens [13]

Streptomyces are widely distributed in soil and play an important ecological role in the biodegradation of insoluble biomaterials through their broad range of extracellular hydrolytic enzymes [17, 24]. Streptomyces are known to decompose chitin, the second most-abundant biomass in nature, and possess many genes for various chitinases with different enzymatic characteristics. Moreover, according to environmental conditions, Streptomyces exhibit morphological differentiation accompanied by the formation of spores and the production of a wide range of secondary metabolites, such as antibiotics $[14,19]$. In fact, Streptomyces are important producers of approximately two-thirds of all antibiotics and other valuable secondary metabolites found so far $[15,16]$.

As saprophytic soil bacteria, Streptomyces utilize naturally occurring polysaccharides, such as chitin, xylan, and cellulose, as carbon sources $N$-Acetylglucosamine (GlcNAc), the monomer of chitin, is a preferred carbon and nitrogen source for Streptomyces, and the related metabolite glutamate is preferred over glucose [18,22]. Chitin is one of the most abundant polysaccharides on earth and is found, among others, in the cuticles and shells of insects and crustaceans and within the cell walls of fungi; to utilize this polysaccharide, Streptomyces have an extensive chitinolytic system [20, 21].

The project was mainly aimed to isolate and identify the predominant fungal strains from the banana plantation and to screen for the potential biocontrol agent against the pathogen. And mainly the study was designed to screen for the possible cooperative role of both the strains (Streptomyces coelicolor, Streptomyces halstedii) towards the fungal pathogen. To confirm the antagonistic we designed to measure the Chitinase activity, biofilm formation and scavenging activity quantitatively in relation to both the species separately and together. Thin layer chromatography was done to screen the antioxidants.

\section{Materials \& Methods}

\subsection{Microorganisms and Culture Condition}

Sample Collection: The samples have been collected from banana plantations in horticulture lab, GKVK agricultural university and coastal regions of Mypad, Nellore in sterile bags and bottles, labelled and preserved at $4^{\circ} \mathrm{C}$. The samples were serially diluted and plated $\left(10^{-9}\right.$ and $10^{-10}$ dilutions) onto potato dextrose agar plates and incubated at $28^{\circ} \mathrm{C}$ for 4-6 days [1]. The isolated colonies were then stained with lacto phenol cotton blue and observed under microscope for their morphology. The pure colonies are identified according to the protocol described by Benson. 2001 [25].

Bacterial culture: The strains selected for the experiment were ordered from MTCC. The strains Streptomyces coelicolor (M130, MTCC No 8), Streptomyces halstedii (CKM-2, MTCC No 6817). The cultures were revived on their respective selective medium as formulated and standardized by MTCC.

The medium used for Streptomyces halstedii is as follows with incubation at $30^{\circ} \mathrm{C}$ for 4 days (Glucose $4.0 \mathrm{~g}$, Yeast extract $4 \mathrm{~g}$, Malt extract $10 \mathrm{~g}, \mathrm{CaCO}_{3} 2 \mathrm{~g}$ per 1litre). The medium used for Streptomyces coeliocolor is as follows with incubation at $26^{\circ} \mathrm{C}$ for 5 days (Glucose $4.0 \mathrm{~g}$, Yeast extract 4g, Malt extract $10 \mathrm{~g}$ per 1 litre).

Colloidal chitin preparation: Colloidal chitin was prepared according to the protocol described elsewhere [1]. Briefly $12 \mathrm{~g}$ of chitin powder was obtained from the shrimp 
shells and digested with $380 \mathrm{ml}$ of concentrated $\mathrm{HCl}$, and the contents were left at $4^{\circ} \mathrm{C}$ overnight with vigorous stirring. The mixture was added to $4 \mathrm{~L}$ of ice-cold ethanol $95 \%(\mathrm{v} / \mathrm{v})$ with rapid stirring and incubated at $4{ }^{\circ} \mathrm{C}$ overnight. The precipitate obtained was collected by centrifugation at $5000 \mathrm{~g}$ for $20 \mathrm{~min}$ at $4^{\circ} \mathrm{C}$ and subsequently washed with sterile distilled water and made neutral $(\mathrm{pH}$ 7.0)

\subsection{Antagonistic Activity}

Cup plate method: The strains were screened for the antagonistic activity by cup plate method as described by Prapagdee et al, [6]. The isolates were screened for their in vitro antagonism, according to the modified method of Crawford et al. $20 \mu \mathrm{l}$ suspension of spores $\left(10^{6}\right.$ spores $\left./ \mathrm{ml}\right)$ of strain was spotted on one side of a PDA plate and incubated at $28^{\circ} \mathrm{C}$ for 3 days. A mycelial plug of $6.0-\mathrm{mm}$ diameter from 3-days-old of each fungus was cut and transferred to a Streptomyces pregrown PDA plate. The fungal plug alone was placed on uninoculated PDA plates separately as control treatment(C). The radial fungal growth in the direction of the antagonist in both the control and the dual culture plates containing Streptomyces halstedii and Streptomyces coelicolor (SHC) was measured at 4 and 6 days after incubation respectively.

Tube Method: Briefly, to a test tube with $10 \mathrm{ml}$ of medium (with $1 \%$ Chitin) $20 \mu$ of culture was added and incubated $37^{\circ} \mathrm{C}$ overnight. The tube without the inoculum was maintained as control.

Crude enzyme extraction: Both the strains (S.coelicolor, S.halstedii) were cultivated in about $200 \mathrm{ml}$ of Streptomyces broth and incubated at $28^{\circ} \mathrm{C}$ in an orbital shaker (200rpm) for 10 days. The culture was centrifuged at $11,000 \mathrm{rpm}$ for $15 \mathrm{~min}$, and the supernatant was filtered with filter paper. The supernatant was used for extraction with n-hexane, ethyl acetate, chloroform and n-butanol. The extracts were then concentrated by evaporation at room temperature and the concentrated products kept at $-20^{\circ} \mathrm{C}$ until further use [23].

Chitinase activity: Chitinase activity was measured with colloidal chitin as a substrate. Briefly, the reaction mixture containing $0.5 \mathrm{ml}$ of $1 \%(\mathrm{w} / \mathrm{v})$ colloidal chitin and $0.5 \mathrm{ml}$ of the crude enzyme was incubated at $50^{\circ} \mathrm{C}$ for 1 hour. The reaction was stopped by the addition of $3 \mathrm{ml}$ of DNS followed by heating at $100^{\circ} \mathrm{C}$ for $5 \mathrm{~min}$. Following centrifugation, the concentration of the reducing sugar in the supernatant was determined using the modified DNS method (Miller, 1959). The absorption of the sample was measured at $530 \mathrm{~nm}$ using a UV spectrophotometer (Shimadzu). One unit (U) of chitinase activity was defined as the amount of enzyme required to produce $1 \mathrm{mM}$ of reducing sugar per min.

The amount of protein in the crude enzyme was measured by the method of Lowry et al, 1951 [26] with BSA as standard.

Biofilm Formation: Biofilm formation studies in the presence of glucose are carried out according to the protocol as described by Arana et al,[10]. Briefly, the cells grown overnight in trypticase soya broth (TSB) $(0.25 \%$ glucose) at $37^{\circ} \mathrm{C}$ are diluted 1:40 with TSB using 0.25 glucose. $200 \mu \mathrm{l}$ of this culture was inoculated into 96well sterile plate followed by $24 \mathrm{hrs}$ incubation at $37^{\circ} \mathrm{C}$. After the incubation the wells were washed thrice with PBS and dried in an inverted position. The culture wells were then stained with $1 \%$ crystal violet for $15 \mathrm{~min}$ and the wells rinsed with $200 \mu \mathrm{l}$ of ethanol: Acetone $(80: 20)$ to solubilise the crystal violet. Absorbance was measured at 595nm. The reaction was set up for all the groups Control, Streptomyces coeliclor, Streptomyces halstedii, and Culture containing both the strains (C, SC, SH, SHC).

Scavenging Activity: The principle of DPPH method is based on the reduction of DPPH in the presence of a hydrogen donating antioxidant. Extracts reduce the colour of DPPH due to the power of hydrogen donating ability [15]. DPPH is one of the compounds that possess a proton free radical with a characteristic absorption, which decreases significantly on exposure to proton radical scavengers.

The DPPH free radical scavenging activity was done on all the groups of experiments (Control, SC, SH and SHC). The cultures with the medium were incubated along with the fungal cultures for 5 days in an incubator at $30^{\circ} \mathrm{C}$. After the incubation, the culture was centrifuged and the supernatant collected. To the supernatant, equal volumes of methanol was added and kept in the orbital shaker overnight. Four tubes were used in the experimentation. First tube serves as the control, with no bacterial cultures. Second tube with Streptomyces coelicolor alone (SC), third tube with Streptomyces halstedii alone (SH), and the fourth tube with both the cultures (SHC). The antioxidants if at all present were expected to be extracted into the methanol medium. The culture supernatant $(150 \mu l)$ of was mixed with $37.5 \mu \mathrm{l}$ methanolic solution containing $0.75 \mathrm{mM} 1,1$ diphenyl-2-picrylhydrazyl (DPPH) (HiMedia, Mumbai, India) radicals. The mixture was shaken vigorously and left to stand for $30 \mathrm{~min}$ in the dark. Absorbance was then measured at $517 \mathrm{~nm}$ against a blank. Ascorbic acid (0.5M) was taken as the positive control. The scavenging ability was calculated as follows: Scavenging ability $(\%)=(\Delta$ Control $-\Delta$ Sample) $/ \Delta$ control $\times 100$.

Thin Layer Chromatography Analysis: The antioxidant materials of the culture supernatant were analyzed by silica gel TLC using 5:4:3 (v/v/v) n-butanol/methanol/16\% aqueous ammonia as the mobile phase [24].

The samples (about 10 $\mu \mathrm{l}$ ) extracted in the methanol were spotted onto Silica gel TLC plates $(0.25 \mathrm{~mm})$. The plate was placed in the chamber saturated with the mobile phase (5:4:3 (v/v/v) n-butanol/methanol/16\% aqueous ammonia) and allowed to run along with the mobile phase. The compounds were then visualized by spraying with ethanol containing $0.5 \%(\mathrm{w} / \mathrm{v})$ Ninhydrin followed by activating on heating in hot air oven at $55^{\circ} \mathrm{C}$. The antioxidant material was identified based on the $\mathrm{Rf}$ value in comparison with standard chitooligosaccharides (HiMedia). 


\section{Results and Discussion}

Isolation and Identification of the fungal pathogen:
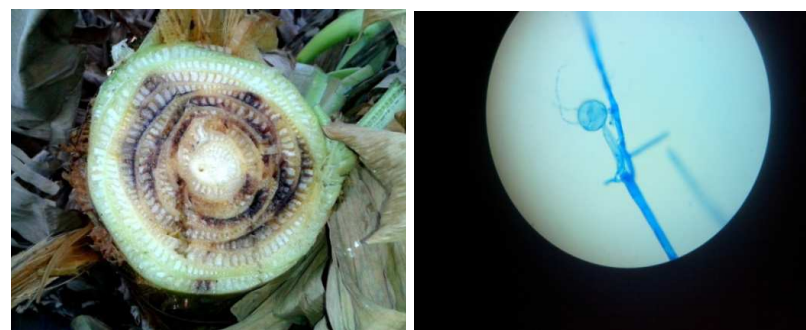

Fig 1. Left: Sample obtained showing Streaks of fungal infection. Right: Microscopic examination of Nigrospora.

\subsection{Revival of Cultures}

The lyophilized cultures were carefully retrieved by inoculating into Streptomyces medium formulated and standardized by MTCC (fig 2).
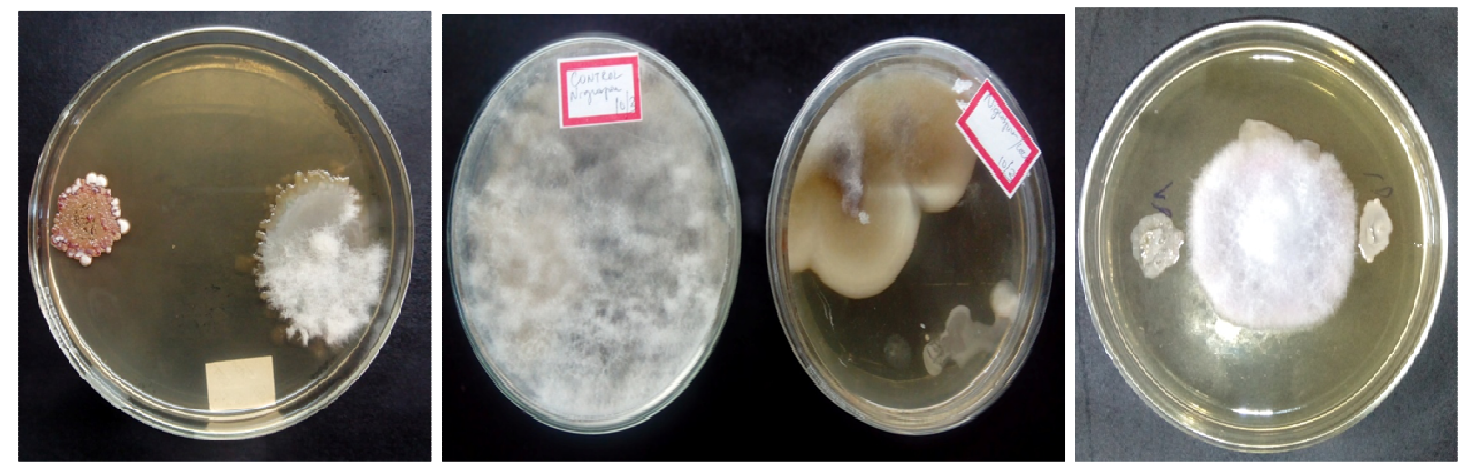

Fig 3. Left: Figure showing the antagonistic activity of the Streptomyces coelicolor against the fungal pathogen Nigrospora. Middle: Antagonistic activity of the Streptomyces halstedii. Right: Antagonistic activity of the both the Streptomyces species against the fungal pathogen Nigrospora.
The bacterial strains were already proved to have antagonistic activity towards the fungal pathogens [12]. But there was no data published of the antagonistic activity confirm our study of the possible cooperative role of both the species we have studied the antagonistic activity on the same plate with both the species.

\subsection{Chitinase Activity}

The extract of $\mathrm{n}$ butanol was found to more effective. So the chitinase activity was carried with the $\mathrm{n}$ butanol extract. Chitinase activity was measured with colloidal chitin as a substrate. The absorption of the appropriately diluted test sample was measured at $530 \mathrm{~nm}$ using a UV spectrophotometer (Beckman DU530, USA) along with substrate and enzyme blanks. One unit (U) of chitinase activity was defined as the amount of enzyme required to produce $1 \mathrm{mM}$ of reducing sugar per min.

The activity of chitinase was observed for all the groups. The chitinase activity was shown in both the species. As per the objective of the study both the cultures were studied together for the chitinase activity in the presence of the pathogen. The chitinase activity was found to be more in the presence of both the cultures together. This reveals of towards the Nigrospora, a common plant pathogen. To
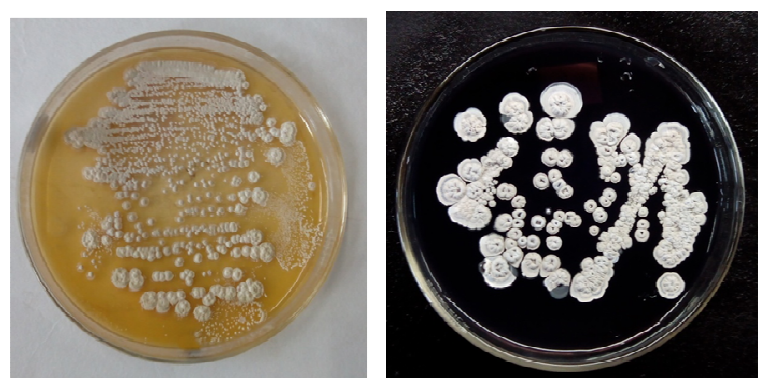

Fig 2. Left: Streptoyces hastedii Right: Streptomyces coeliocolor

\subsection{Screening for the Antagonistic Activity}

The fungal plug was additionally placed on uninoculated PDA plates separately as control treatment. The radial fungal growth in the direction of the antagonist in both the control and the dual culture plates was measured at 4 and 6 days after incubation respectively. the presence of the possible role of both the species towards the antagonistic activity of the Nigrospora species.

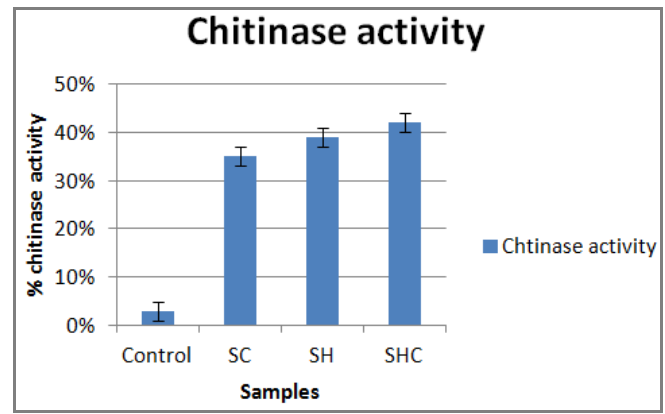

Fig 4. Graph shwoing the \% chitinase activity of the samples Control, SC, $\mathrm{SH}$ and SHC. All the values were average of triplicates. The values are expressed in \%. SC: Streptomyces coelicolor, SH: Streptomyces halstedii, SHC: Both the species.

\subsection{Biofilm Formation}

The biofilm studies confirms of the cooperative role of the species. Streptomyces species exhibits biofilms. The increase or stability in the values proves of the possible cooperative role of both the species in the antagonistic activity against the Nigrospora species. A one way ANOVA 
was done to show the significance in the formation of biofilms. There was a significant effect in the formation of the biofilms between the groups remembered at the $p<$
0.05 level. $[F(3,11)=47.855 .272, p=0.0022]\left[\mathrm{F}_{\text {crit }}\right.$ value being 4.0661].

Table 1. Table showing the values of biofilm formation after ethanol washing. All the values are average of triplicates. All the values are expressed as value \pm sd. Control: Medium alone; SC: Streptomyces coeliclor; SH: Streptomyces halstedii.

\begin{tabular}{lllll}
\hline BIOIFILM formation & Control & SC & SH & SC+SH \\
\hline & 0.114 & 0.315 & 0.293 & 0.383 \\
O.D at 595nm & 0.123 & 0.341 & 0.277 & 0.391 \\
& 0.119 & 0.318 & 0.283 & 0.397 \\
Mean & $0.119 \pm 0.0045$ & $0.325 \pm 0.0142$ & $0.284 \pm 0.008$ & $0.390 \pm 0.007$ \\
\hline
\end{tabular}

\subsection{DPPH Free Radical Scavenging Activity}

All the four groups showed a positive effect of antioxidant activity. The control (Fungus and medium) also showed antioxidant activity. This confirmed of the presence of the defense mechanism of the fungus. The $\mathrm{SC}, \mathrm{SH}$ and SHC showed the scavenging activity. All the groups showed the similar activity when compared with the positive control. The O.D of the DPPH plus the methanol taken as the control was found to be 1.012 .

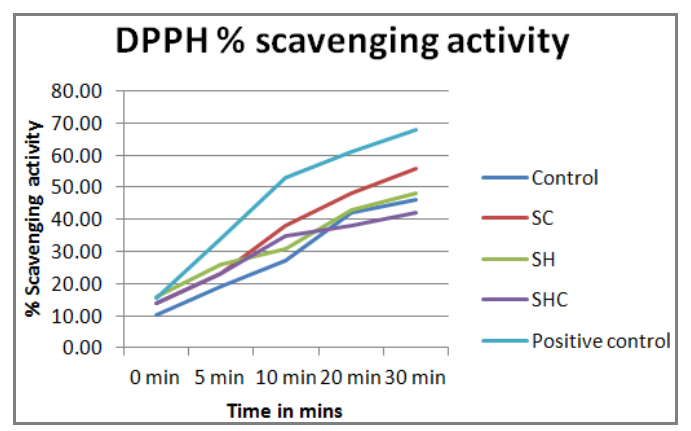

Fig 5. Table showing the percent free radical scavenging activity of the groups. Ascorbic acid was taken as the positive control. All the values were average of triplicates. (SC: Streptomyces coeliclor; SH: Streptomyces halstedii; SHC: Both the species together).

\subsection{Thin Layer Chromatography}

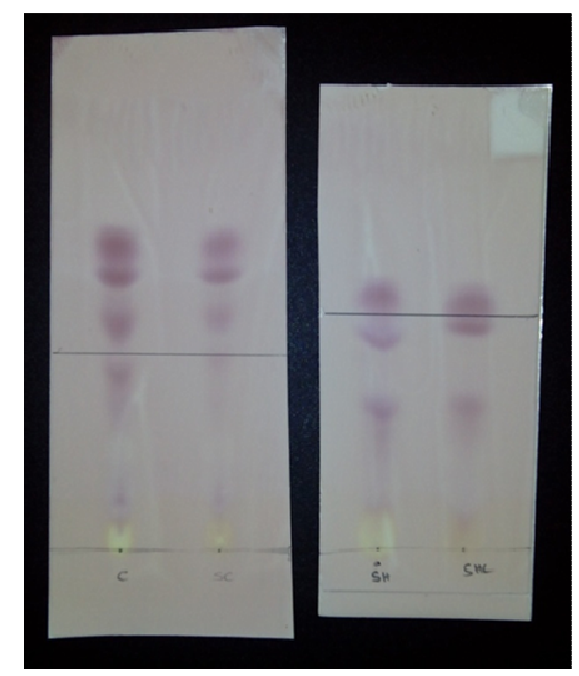

Fig 6. TLC analysis of antioxidant materials in the culture supernatant of the experimental groups (Control, SC, SH and SHC). After developing, the compounds were then visualized by spraying with ethanol containing $0.5 \%$ $(w / v)$ Ninhydrin.
The results obtained in the TLC were calculated for the $\mathrm{Rf}$ values. The $\mathrm{Rf}$ values obtained were compared with the standard antioxidant $\mathrm{Rf}$ values. The $\mathrm{Rf}$ values of 0.12 match with the catechin, and 0.4 match with the Quercetin, 06 match with caffeic acid. The culture flask containing both the species showed an inhibition of the compounds of Rf values 0.6 and 0.68 .

Table 2. Table showing the Rf values of the Antioxidant compounds resolved on TLC plate.

\begin{tabular}{lllll}
\hline & Control & SC & SH & SHC \\
\hline \multirow{4}{*}{ Rf values } & 0.12 & 0.12 & 0.12 & 0.12 \\
& 0.4 & 0.5 & 0.31 & 0.31 \\
& 0.5 & 0.6 & 0.45 & 0.5 \\
& 0.6 & 0.68 & 0.57 & 0.57 \\
\hline
\end{tabular}

\section{Discussion}

The Actinomycetes were already proved to have antagonistic activity towards the fungal pathogens [12]. But there was no data published of the antagonistic activity towards the Nigrospora, a common plant pathogen. To confirm our study of the possible cooperative role of both the species we have studied the antagonistic activity on the same plate with both the species. The plate result also confirms of the activity. The activity of chitinase was observed for all the groups. The chitinase activity was shown in both the species [21]. As per the objective of the study both the cultures were studied together for the chitinase activity in the presence of the pathogen. The chitinase activity was found to be more in the presence of both the cultures together. This reveals of the presence of the possible role of both the species towards the antagonistic activity of the Nigrospora species.

The biofilm studies confirms of the cooperative role of the species. Streptomyces species exhibits biofilms. Previous literature also showed of the exhibition of biofilm formation of the Streptomyces species [15]. The increase or stability in the values proves of the possible cooperative role of both the species in the antagonistic activity against the Nigrospora species. A one way ANOVA was done to show the significance in the formation of biofilms. All the four groups showed a positive effect of antioxidant activity. The control (Fungus and medium) also showed antioxidant activity. This confirmed of the presence of the defense mechanism of the fungus. All the groups ( $\mathrm{SC}, \mathrm{SH}$ and $\mathrm{SHC}$ ) 
showed the similar activity when compared with the positive control.

Peculiar results were obtained in the TLC. The Rf values obtained were compared with the standard antioxidant $\mathrm{Rf}$ values. The $\mathrm{Rf}$ values of 0.12 match with the Catechin, and 0.4 match with the Quercetin, 06 match with Caffeic acid. The culture flask containing both the species showed an inhibition of the compounds of $\mathrm{Rf}$ values 0.6 and 0.68 .

\section{Summary}

Microbial antagonists are widely used for the biocontrol of fungal plant diseases. Since chitin is the major component of most fungal cell walls, a principal role has been attributed to enzymes from the chitinolytic system. Enzymatic lysis of fungal cell walls through extracellular chitinases has been implicated as a mechanism of biocontrol by bacterial agents. Streptomyces are found predominantly in soil and decaying vegetation and are the main decomposers of chitin. The expression of five $c h i$ genes (chiA, chiB, chiC, chiD, and $c h i F)$ is induced by chitin in the bacterium.

The Streptomyces have already been proved of the antagonistic activity against the fungal pathogens. But there were no published data on the Nigrospora species. For the first time we try to show the possible cooperative role of the two Streptomyces species towards the antagonistic activity. The experimental objectives of Biofilm formation, Scavenging activity and Anatagonistic chitinolytic activity all proved of the cooperative role of the two species. The antioxidants released also showed of the inhibitory effect of the compounds. These compounds need to be isolated in pure form to fight against the fungal pathogens and protect the crop, which might be more beneficial to the farmers as it is cost effective when compared to the chemicals. Moreover it is natural and reduces the use of harmful chemical fungicides.

\section{References}

[1] M Zarei, S Aminzadeh, H Zolgharnein, A Safahieh, M Daliri, K A Noghabi, A Ghoroghi, A Motallebi, 2011, Characterization Of A Chitinase With Antifungal Activity From A Native Serratia Marcescens B4a, Brazilian Journal of Microbiology 42: 1017-1029

[2] AL Garda, JMF Abalos, P Sanchez, A R Arribas, RI Santamaria, 1997, Two genes encoding an endoglucanase and a cellulose-binding protein are clustered and coregulated by a TTA codon in Streptomyces halstedii JM8. Biochem J 324:403-411.

[3] G Duan, N Christian, J Schwachtje, D Walther, and O Ebenhöh, 2013. The Metabolic Interplay between Plants and Phytopathogens, Metabolites, 3, 1-23.

[4] G Vaaje-Kolstad, S J Horn, M. F Daan, B Synstad, and Vincent G. H. Eijsink 2005, The Non-catalytic Chitinbinding Protein CBP21 from Serratia marcescens Is Essential for Chitin Degradation J. Biol. Chem. 2005, 280:28492-28497.
[5] B Nazari, M Kobayashi, A Saito, A Hassaninasa, K Miyashita, T Fujiia 2013, Chitin-Induced Gene Expression in Secondary Metabolic Pathways of Streptomyces coelicolor A3(2) Grown in Soil. Applied and Environmental Microbiology p. Volume 79, 707-713.

[6] B Prapagdee, C Kuekulvong and S Mongkolsuk, 2008, Antifungal Potential of Extracellular Metabolites Produced by Streptomyces hygroscopicus against Phytopathogenic Fungi, Int. J. Biol. Sci., 4. 330-337.

[7] B. B. Aam, E. B. Heggset, A. L Norberg, M. S. Kjell, M. Varum and V. G. H. Eijsink, 2010, Production of Chitooligosaccharides and their potential Applications in Medicine, Mar. Drugs, 8, 1482-1517

[8] C Shi, P Yan, J Li, H Wu, Q Li and S Guan 2014. Biocontrol of Fusarium graminearum Growth and Deoxynivalenol Production in Wheat Kernels with Bacterial Antagonists, Int. J. Environ. Res. Public Health, 11, 1094-1105.

[9] M. A Hannan, M. M. Hasan, I. Hossain1, S. M. E. Rahman, A M Ismail, and Deog-Hwan Oh, 2012, Integrated Management of Foot Rot of Lentil Using Biocontrol Agents under Field Condition. J. Microbiol. Biotechnol., 22(7), 883-888.

[10] A. T. Arana, J. Valle, C. Solano, M.A Jesu' S Arrizubieta, C Cucarella, M Lamata, B. Amorena, J. Leiva, Jose' R. Penade, And In Igo Lasa, 2001, The Enterococcal Surface Protein, Esp, Is Involved in Enterococcus faecalis Biofilm Formation, Appl. Environ. Microbiol., 67(10):4538.

[11] K.C Hoang, T. H Lai, C. S Lin, Y. T Chen and C. Y Liau, 2011. The Chitinolytic Activities of Streptomyces sp. TH-11, Int. J. Mol. Sci., 12, 56-65.

[12] A. S. Magdalena E. Tenconi,b S. Rigali, and P. Gilles. VWezela 2011. Functional Analysis of the $\mathrm{N}$ Acetylglucosamine Metabolic Genes of Streptomyces coelicolor and Role in Control of Development and Antibiotic Production. Journal of Bacteriology p. 1136-1144.

[13] M. S Brzezinsk U. Jankiewicz., 2012, Production of Antifungal Chitinase by Aspergillus niger LOCK 62 and Its Potential Role in the Biological Control, Curr Microbiol 65:666-672.

[14] R. Nandakumar, S. Babu1, T. Raguchander and R. Samiyappan, 2007, Chitinolytic Activity of Native Pseudomonas fluorescens Strains J. Agric. Sci. Technol. Vol. 9: $61-68$.

[15] T. Kawase, S. Yokokawa, A. Saito; T. Fujii, N. nikaidou, K. Miyashita, and Takeshi Watanabe, 2005. Comparison of Enzymatic and Antifungal Properties between Family 18 and 19 Chitinases from $S$. coelicolor A3(2), Biosci. Biotechnol. Biochem., 70 (4), 988-998, 2006.

[16] P. N. Lipke And R. Ovalle, 1998, MINIREVIEW Cell Wall Architecture in Yeast: New Structure and New Challenges $J$. Bacteriol, 180(15):3735.

[17] R. Hamid, M. A. Khan, M. Ahmad, M. M. Ahmad, M. Z Abdin, J. Musarrat, and S. Javed., 2013. Chitinases: An update J Pharm Bioallied Sci. Jan-Mar; 5(1): 21-29.

[18] S. H Ji, N. C Paul, J. X Deng, Y. S Kim, B. S Yun and S. H $\mathrm{Yu}, 2013$. Biocontrol Activity of Bacillus amyloliquefaciens CNU114001 against Fungal Plant Diseases, Mycobiology December, 41(4): 234-242. 
[19] S. Subramaniam, V. Ravi, G. K Narayanan. 2012. Studies on Production of Enzyme Chitinase from Streptomyces sp. and its anti-fungal activity. Journal of Pharmacy Research,5(3),1409-1413.

[20] T. Watanabe, W. Oyanagi, K. Suzuki, and H. Tanaka, 1990. Chitinase System of Bacillus circulans WL-12 and Importance of Chitinase $\mathrm{Al}$ in Chitin Degradation, J. Bacteriol. vol. 172 no. 74017-4022.

[21] S. Rigali, F. Titgemeyer, S. Barends, S. Mulder, A. W. Thomae, D. A Hopwood \& G. P. van Wezel 2008. Feast or famine: the global regulator DasR links nutrient stress to antibiotic production by Streptomyces, EMBO reports, 9, 670-675.

[22] D.L Crawford, J.M Lynch, J.M Whipps, M.A Ousley (1993). Isolation and characterization of Actinomycete antagonists of a fungal root pathogen. Appl. Environ. Microbiol. 59: 3899-3905.
[23] Sutthinan Khamna, Akira Yokota, John F Peberdy, Saisamorn Lumyong 1, 2009. Antifungal activity of Streptomyces spp. isolated from rhizosphere of Thai medicinal plants, International Journal of Integrative Biology. Vol. 6, No. 3, 143.

[24] K. Kadokura , A. Rokutani, M. Yamamoto, T. Ikegami, H. Sugita, S. Itoi, W. Hakamata, T. Oku, T. Nishio. 2007. Purification and characterization of Vibrio parahaemolyticus extracellular chitinase and chitin oligosaccharide deacetylase involved in the production of heterodisaccharide from chitin. Appl Microbiol Biotechnol. 75: 357-365.

[25] J. H. Benson. Microbiological Applications : Laboratory Manual in General Microbiology, Short Version, 8th Edition; Published by McGraw Hill.

[26] O. H Lowry, N.J Rosebrough, A.L Farr, R. J Randall (November 1951). "Protein measurement with the Folin phenol reagent". J. Biol. Chem. 193 (1): 265-75. 